\title{
Large-Scale, Long-Term Stable Femtosecond Timing Distribution and Synchronization Systems
}

\author{
Jungwon Kim, Frank Ludwig*, Jeff Chen, Florian Loehl*, Franco Wong, Holger Schlarb*, and Franz Kärtner \\ Department of Electrical Engineering and Computer Science, Research Laboratory of Electronics, \\ Massachusetts Institute of Technology, Cambridge, MA 02139, USA. \\ *Deutsches Elektronen-Synchrotron, Hamburg, Germany.
}

\begin{abstract}
We demonstrate large-scale, long-term stable, and femtosecond-precision timing distribution and synchronization systems based on optical pulse trains generated from ultralownoise mode-locked lasers.
\end{abstract}

\section{SYSTEM OVERVIEW}

Mode-locked lasers have a great potential for generating ultra low-jitter optical and rf-signals, and serving as optical master oscillators for large-scale, high-precision timing distribution and synchronization systems. Fig. 1 shows the conceptual diagram for the envisioned timing system based on mode-locked lasers. The optical pulse trains are generated from mode-locked lasers and delivered by timing stabilized fiber links to all remote locations where precise timing is required. At these locations, either local laser systems or rfsources are tightly synchronized to the output pulse trains from the timing link.

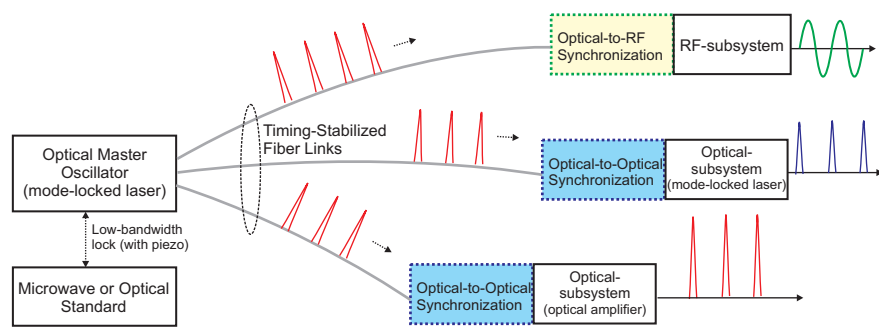

Fig 1. Schematic outline of timing distribution and synchronization system.

Large-scale, high-precision timing systems are crucial for the seeded x-ray free-electron lasers [1], phased-array antennas [2], and the transfer of precise time and frequency information over long distances [3]. One of the biggest challenges in the implementation of such timing systems is attaining long-term drift-free operation with femtosecond (and potentially subfemtosecond) precision. In this paper, we propose and demonstrate key components and techniques that enable long-term stable femtosecond precision.

\section{MOde-LOCKed LASER AS A MASTER OSCILlator}

The use of mode-locked lasers as optical master oscillators has many advantages [4]: (i) any harmonic components of the fundamental repetition rate can be recovered; (ii) the group delay of the fiber link is directly stabilized; (iii) optical crosscorrelation can be easily employed for link stabilization as well as optical-to-optical synchronization; and (iv) the pulse train can directly seed optical amplifiers.
To minimize the undesired impact from fiber nonlinearities, it is highly desirable to use a high repeptition-rate laser, preferably a few hundreds MHz. The timing jitter integrated from $10 \mathrm{kHz}$ to Nyquist bandwidth should be minimal, preferable well below $10 \mathrm{fs}$. In addion, the pulsewidth should be reasonably short, preferably hundreds fs, to enable efficient optical cross-correlation. As a representative example of such a laser, recently a 194-MHz repetition rate soliton Er-fiber laser was developed [5]. The use of gain media with long upper-state lifetime such as Er enables low timing jitter and amplitude noise in the high frequency range. The measured relative intensity noise and timing jitter integrated from 10 $\mathrm{kHz}$ to $10 \mathrm{MHz}$ are $0.003 \%$ and $10 \mathrm{fs}$, respectively. The measured pulsewidth is $167 \mathrm{fs}$. We used this laser for the timing link stabilization presented in Sec. III.

\section{Timing StabiLIZEd Fiber LinKS}

Once a low-noise pulse train is generated from the master oscillator, it should be disseminated to remote rf- and optical subsystems that we aim to synchronize. In doing so, acoustic noise and thermal drifts introduced to the fiber link should be properly canceled. The timing stabilization of the fiber links is achieved by (1) partial back-reflection of the delivered pulse train at the remote location, (2) detecting the timing error between the back-reflected pulses and the fresh pulses from the laser, and (3) using this error signal to compensate undesired timing fluctuation by the counteraction of a piezostretcher when a feedback loop is closed. Now the main challenge is how to implement a high-resolution and drift-free timing detector between back-reflected and fresh pulses. Conventional techniques based on microwave mixers suffer from thermal drifts, and showed a limited long-term stability.

To overcome these limitations, we developed a new singlecrystal, self-aligned balanced optical cross-correlator for high resolution and drift-free detection of timing error [6]. Fig. 2(a) shows the schematic of the balanced cross-correlator based on a type-II phase-matched PPKTP crystal. The PPKTP crystal combines the second-harmonic generation (SHG) and the walk-off between orthogonally polarized pulses. This enables the balancing of the cross-correlator at the same center wavelength. The measured SHG efficiency of the PPKTP crystal at $1550 \mathrm{~nm}$ was $\sim 0.4 \%$, which provides enough SHG to be detected with commercially available photodetectors.

Fig. 2(b) shows the long-term out-of-loop timing drift trace over $100 \mathrm{~s}$, when a 310 -meter long fiber link is stabilized. The measured timing jitter $[10 \mathrm{mHz}, 100 \mathrm{kHz}]$ is $9.7 \mathrm{fs}$, where the 
photodetector background noise corresponds to 8.2 fs. Note that the fiber link is not environmentally well-protected, and the displacement in $100 \mathrm{~s}$ is already comparable to the piezostretcher's range $(\sim 0.7 \mathrm{~mm})$. With an additional motorized translation stage with longer range, it will be possible to maintain this level of accuracy as long as desired. (a)

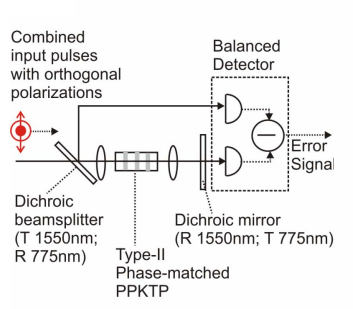

(b)

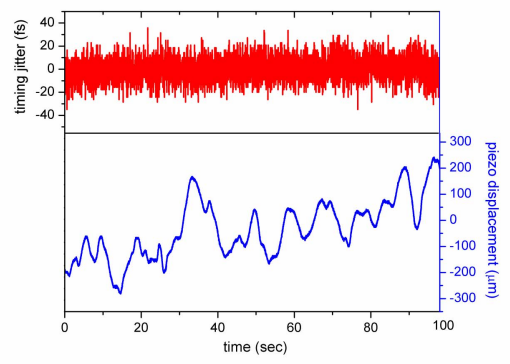

Fig. 2. (a) Schematic of the single-crystal balanced cross-correlator for timing link stabilization. (b) Long-term out-of-loop timing jitter and piezo-stretcher displacement over 100 seconds. Measured timing jitter is $9.7 \mathrm{fs}$, where the detector background noise corresponds to $8.2 \mathrm{fs}$.

\section{OPTICAL-TO-RF SYNCHRONIZATION}

Although we can deliver ultralow-noise pulse trains to remote locations via timing stabilized fiber links, it is a highly nontrivial task to transfer the low noise properties of the pulse train in the optical domain to the electronic domain, and extract a drift-free, ultralow-jitter rf-signal. The extraction of an rf-signal from an optical pulse train using direct photodetection suffers from excess phase noise where the major origin is the amplitude-to-phase conversion in the photodetectors and microwave mixers [7]. Although careful suppression of excess noise enabled to demonstrate short-term $0.8 \mathrm{fs}$ jitter [8], it is still challenging to keep the phase stability of the extracted rf-signal in a long-term drift-free manner.

To circumvent amplitude-to-phase conversion and to ensure long-term stable rf-signal extraction, a balanced opticalmicrowave phase detector [9] was recently proposed and demonstrated. It is based on the precise phase detection in the optical domain using a differentially-biased Sagnac fiber loop and synchronous detection. By detecting the phase error between pulse trains and rf-signals with electro-optic sampling in the optical domain, it is robust against thermal drifts and photodetector nonlinearities. In addition, one can leverage the fly-wheel effect of VCOs to achieve both low noise performance and high enough output power levels $(>10 \mathrm{dBm})$.

To test the performance of this phase detector, we measured the out-of-loop phase drift between two optoelectronic phaselocked loops (PLLs) using two of these phase detectors. The short-term performance is 12.8 fs out-of-loop relative jitter [10Hz, $10 \mathrm{MHz}]$ between the two $10.225 \mathrm{GHz}$ rf-signals at $+10 \mathrm{dBm}$ power level. As shown in Fig. 3(a), the long-term drift in the extracted rf-signals is within 48 fs over $1 \mathrm{~h}$ time scale. Note that the long-term measurement was limited by the drift of the mixer and electronic amplifiers used in the out-ofloop characterization setup itself, as shown in Fig. 3(b). Even the temperature of the characterization setup is actively stabilized, drifts up to $41 \mathrm{fs}$ (in $1 \mathrm{~h}$ ) and $48 \mathrm{fs}$ (in $4 \mathrm{~h}$ ) are observed at certain time frames. Further efforts to eliminate electronic noise sources and to reach shot-noise limited detection are in progress. To overcome the limitations of longterm measurement, we are currently investigating the use of an all-optical technique for drift-free timing detection.
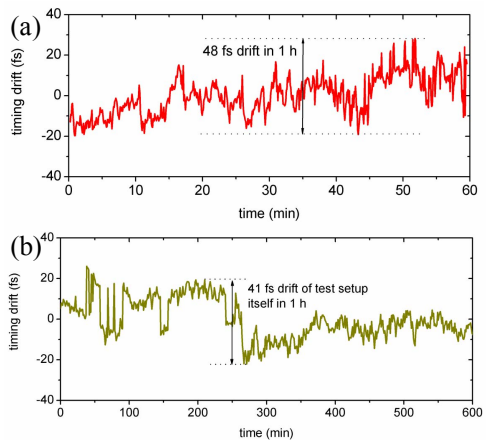

(c)

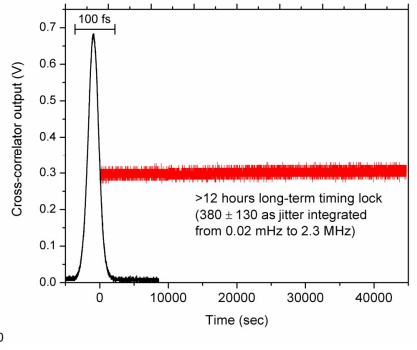

Fig. 3. (a) Long-term out-of-loop drift measurement between two locked VCOs shows that timing drift is within $48 \mathrm{fs}$ in $1 \mathrm{~h}$. (b) Long-term baseline measurement of the characterization setup. The same 10-GHz signal is applied to both inputs of the mixer. Timing drifts up to $41 \mathrm{fs}$ (in $1 \mathrm{~h}$ ) and $48 \mathrm{fs}$ (in $4 \mathrm{~h}$ ) are observed. (c) Long-term out-of-loop cross-correlation between locked Ti:sapphire and Cr:forsterite lasers over $12 \mathrm{~h}$. The measured jitter is 380 as integrated from $0.02 \mathrm{mHz}$ to $2.3 \mathrm{MHz}$ [11].

\section{OPTICAL-TO-OPTICAL SYNCHRONIZATION}

Tight synchronization is necessary not only between optical and rf-systems but also between different optical systems. To achieve long-term stable optical-to-optical synchronization, a balanced cross-correlator can be used. Previously, we demonstrated a balanced cross-correlator based on group delay dispersion (GDD) for long-term sub-fs synchronization of two independently mode-locked lasers operating at different center wavelength $[10,11]$. Fig. 3(c) shows the out-of-loop crosscorrelation trace between locked Ti:sapphire and Cr:forsterite lasers over $12 \mathrm{~h}$. The timing jitter is 380 as \pm 130 as integrated from $0.02 \mathrm{mHz}$ to $2.3 \mathrm{MHz}$ [11]. For synchronization of two lasers at the same center wavelength, the single-crystal balanced cross-correlator in Sec. III can be easily employed.

\section{SUMMARY}

We reported the progress and preliminary results toward long-term stable and scalable timing distribution and synchronization systems. We developed a single-crystal balanced cross-correlator and stabilized 310-m fiber links in 10 -fs precision. A balanced optical-microwave phase detector is used to regenerate rf-signals within $<50$ fs drift in $1 \mathrm{~h}$, where the accuracy was mainly limited by the measurement setup itself. Finally, sub-fs optical synchronization over $12 \mathrm{~h}$ was demonstrated using a balanced cross-correlator.

\section{REFERENCES}

[1] J. Kim et al., FEL 2004, p. 339, August 2004.

[2] B. Shillue, LEOS Summer Topical Meetings 2005, p. 61, July 2005.

[3] S. Foreman et al., Rev. Sci. Instrum. 78, 021101 (2007).

[4] F. X. Kärtner et al., PAC 2005, p. 284, May 2005.

[5] J. Chen et al., Paper CThHH3, to be presented at CLEO 2007, May 2007.

[6] J. Kim et al, Opt. Lett. 32, 1044 (2007).

[7] E. N. Ivanov et al., IEEE Trans. UFFC 52, 1068 (2005).

[8] J. J. McFerran et al, Electron. Lett. 41, 650 (2005).

[9] J. Kim et al., Opt. Lett. 31, 3659 (2006).

[10] T. R. Schibli et al, Opt. Lett. 28, 947 (2003)

[11] J. Kim et al, EPAC 2006, p. 2744, June 2006. 